\title{
High failure rate of the interspinous distraction device (X-Stop) for the treatment of lumbar spinal stenosis caused by degenerative spondylolisthesis
}

\author{
Olaf J. Verhoof · Johannes L. Bron • \\ Frits H. Wapstra · Barend J. van Royen
}

Received: 13 February 2007/Revised: 6 July 2007/ Accepted: 17 August 2007/Published online: 11 September 2007

(c) Springer-Verlag 2007

\begin{abstract}
The X-Stop interspinous distraction device has shown to be an attractive alternative to conventional surgical procedures in the treatment of symptomatic degenerative lumbar spinal stenosis. However, the effectiveness of the X-Stop in symptomatic degenerative lumbar spinal stenosis caused by degenerative spondylolisthesis is not known. A cohort of 12 consecutive patients with symptomatic lumbar spinal stenosis caused by degenerative spondylolisthesis were treated with the X-Stop interspinous distraction device. All patients had low back pain, neurogenic claudication and radiculopathy. Pre-operative radiographs revealed an average slip of $19.6 \%$. MRI of the lumbosacral spine showed a severe stenosis. In ten patients, the X-Stop was placed at the L4-5 level, whereas two patients were treated at both, L3-4 and L4-5 level. The mean follow-up was 30.3 months. In eight patients a complete relief of symptoms was observed post-operatively, whereas the remaining 4 patients experienced no relief of symptoms. Recurrence of pain, neurogenic claudication, and worsening of neurological symptoms was observed in three patients within 24 months. Post-operative radiographs and MRI did not show any changes in the percentage of slip or spinal dimensions. Finally, secondary surgical treatment by decompression with posterolateral fusion was performed in seven patients (58\%) within 24 months. In conclusion, the X-Stop interspinous distraction device showed an extremely high failure rate, defined as surgical re-
\end{abstract}

O. J. Verhoof · J. L. Bron · F. H. Wapstra .

B. J. van Royen $(\square)$

Department of Orthopaedic Surgery,

VU University Medical Center,

P.O. Box 7057, 1007 MB Amsterdam,

The Netherlands

e-mail: BJ.vanRoyen@vumc.nl intervention, after short term follow-up in patients with spinal stenosis caused by degenerative spondylolisthesis. We do not recommend the X-Stop for the treatment of spinal stenosis complicating degenerative spondylolisthesis.

Keywords Lumbar spinal stenosis - X-Stop ·

Degenerative spondylolisthesis

\section{Introduction}

Lumbar spinal stenosis complicating degenerative spondylolisthesis is a common cause for low back pain, neurogenic claudication, and radiculopathy in the elderly population. The majority of the patients will respond well to non-operative treatment modalities. However, in patients that fail to respond to conservative treatment, surgical decompression with or without a posterolateral fusion and instrumentation, may be considered [3, 12]. Unfortunately, these procedures have variable long-term outcomes and are frequently followed by complications, especially in the elderly patients with high co-morbility $[2,10]$. Therefore, alternative therapies are being developed, of which the interspinous distraction device is rapidly gaining popularity $[4,9]$. Of such, the X-Stop (X-Stop, St. Francis Medical Technologies, Inc ${ }^{\circledR}$, Alameda, CA) has been introduced as a minimal invasive surgical procedure to treat symptomatic degenerative lumbar spinal stenosis [4, 9]. Initial results of the treatment of degenerative lumbar spinal stenosis with the X-Stop are promising $[8,13,14]$. Recently, encouraging results have been reported for the treatment of patients with symptomatic lumbar spinal stenosis caused by degenerative spondylolisthesis [1]. However, we observed an alarmingly high failure rate, defined as surgical re-intervention, in a cohort of patients treated with the 
X-Stop for symptomatic lumbar spinal stenosis caused by degenerative spondylolisthesis. This prompted us to perform a retrospective chart review, and analysis of the radiographs.

\section{Patients and methods}

We retrospectively reviewed 12 consecutive patients with symptomatic lumbar spinal stenosis caused by degenerative spondylolisthesis treated with the X-Stop interspinous distraction device. The patients were treated between January 2003 and May 2005. There were 9 female and 3 male patients with a mean age at surgery of 67.5 years (50-83). All patients complained of progressive low back pain throughout the day with neurogenic claudication, radiculopathy and a diminished walking distance. In all patients, neurological examination was judged normal or nonspecific. Anteroposterior, lateral and flexion/extension plain radiographs, and magnetic resonance imaging (MRI) were performed in all cases. The percentage of degenerative slip was measured on the lateral radiograph and measured according to the method described by Anderson et al. [1]. The anteroposterior dural sac diameter in the axial and sagittal plane T2 sequence was measured on the MRI. A standardized walking and cycling test [6] was performed at the department of physical therapy. A limited walking distance less than $1 \mathrm{~km}$ ( 0.62 miles) independent of the time needed, was considered positive. After walking the patient had to sit and the pre-walking pain must be reduced in less than $5 \mathrm{~min}$. Cycling should be unlimited without complaints. Initial treatment consisted on patient education, medications to control pain, and exercise and physical treatments to regain or maintain activities of daily living. Surgical treatment with the X-Stop was considered in patients not improving with conservative care for more than 6 months.

All operations were performed under general anesthesia. The patients were placed in prone position on a Wilson spinal surgery frame (Orthopaedic Systems, Inc., Union City, CA) with the lumbar spine in maximum flexion. Prophylactic antibiotics, cefazolin (cefalosporin, Kefzol ${ }^{\mathbb{R}}$ ) $1,000 \mathrm{mg} \mathrm{IV}$, were administered at the induction of anesthesia, and as a second and third dose 8 and $16 \mathrm{~h}$ postoperatively, respectively. After radiographic identification of the surgical level, a mid-sagittal incision of approximately $4 \mathrm{~cm}$ is made over the spinous processes. The musculature was elevated to the level of laminae and facets. The supraspinal ligament is kept intact. To pierce the interspinous ligament, a curved dilator is inserted in the anterior margin of the interspinous space. Subsequently, a sizing distractor is inserted to determine the appropriate implant size. The X-Stop is inserted into the interspinous space as close to the posterior aspect of the lamina as possible. An adjustable wing is attached to the implant and secured along the midline. Patients were mobilized immediately once they had recovered from the anesthetic effects. They were discharged from hospital within $48 \mathrm{~h}$.

Clinical follow-up took place at 6 and 12 weeks and at 12 and 24 months. All patients underwent a clinical and radiographic examination of the lumbar spine in standing position at each follow-up visit. The mean follow-up was 30.3 months (13-41). In patients with persistent or recurrence low back pain with neurogenic claudication and radiculopathy, a second MRI was made. The endpoint was secondary surgical intervention of the lumbar spine. Statistical analysis, comparing the pre- en post-operative MRI dimensions, was performed using Students' $t$-test.

\section{Results}

The pre-operative percentage of degenerative spondylolisthesis was less than $30 \%$ in all patients, with an average slip of $19.6 \% \pm 6.20$ (9.6-29.7). In 9 out of the 12 patients there was a slip of less than $25 \%$ (grade 1) degenerative spondylolisthesis. Bending radiographs revealed mobility at the level of the spondylolisthesis in all patients. MRI showed nerve root compression and impingement of the thecal sac. The mean anteroposterior axial cross-sectional diameter was $7.33 \mathrm{~mm}(5.71-11.19)$ and the mean anteroposterior sagittal cross-sectional diameter was $7.32 \mathrm{~mm}$ (5.40-8.49).

The operations were performed at $\mathrm{L} 4-5$ in ten of the patients and at both L3-4 and L4-5 in two patients. A 14$\mathrm{mm}$ diameter X-Stop was implanted in nine levels. In the remaining levels, a $12-\mathrm{mm}$ implant was used three times, and 16 and 10-mm implants both once. No peri-operative complications were observed. Post-operative plain radiographs showed a correct position of the implants in all patients. No fractures of the spinous processes were observed. The post-operative percentage of spondylolisthesis, measured on plain radiographs post-operatively and at final follow-up, remained unchanged in all patients.

Direct post-operatively, 8 out of 12 patient reported a significant improvement of pain, neurogenic claudication, and radiculopathy. However, four patients did not experience any relief of symptoms following surgery and no improvement at follow-up. At 12 weeks follow up, two patients, that initially had experienced a relief of symptoms suffered from a recurrence of pain, neurogenic claudication, and radiculopathy. In addition, a third patient experienced a recurrence of symptoms at 24 months follow-up.

All patients with persistent or recurrent symptoms had a post-operative MRI. No statistically significant $(P>0.05)$ difference of spinal stenosis was seen at the effected levels 
in comparison to the pre-operative values. The mean postoperative anteroposterior axial cross sectional diameter was $6.80 \mathrm{~mm}(5.24-7.65)$ and the mean sagittal cross sectional diameter was $6.91 \mathrm{~mm}(5.12-7.70)$ (Fig. 1, 2, 3). The pre-operative axial and sagittal cross sectional diameter in these seven patients ( 7 levels) was not significantly different $(P>0.05)$ from that of the five patients ( 7 levels) without persistent or recurrent symptoms. Finally, the seven patients with persistent or recurrent symptoms underwent surgical re-intervention. The mean degenerative spondylolisthesis of these seven patients was $17.8 \% \pm 6.9$. Six of these patients had a pre-operative degenerative spondylolisthesis of less than $25 \%$. One patient had a $27.6 \%$ degenerative spondylolisthesis. The X-Stop was removed and a decompression and posterolateral fusion with instrumentation was performed.

\section{Discussion}

The X-Stop interspinous distraction device has shown to be an attractive alternative to conventional surgical procedures in the treatment of symptomatic degenerative lumbar spinal stenosis $[4,9]$. It may be questioned, however, if the $\mathrm{X}$-stop will be effective in patients with lumbar spinal stenosis caused by degenerative spondylolisthesis also. To our best knowledge, there is only one study that investigated the clinical effects of the X-Stop in patients with lumbar spinal stenosis caused by degenerative spondylolisthesis [1]. In this study, 42 patients were treated with the $\mathrm{X}$-Stop and compared to 33 patients with non-operative treatment. The indication for treatment was a percentage of slip of less than $25 \%$. An overall clinical success rate of 63.4\% was reported in the X-Stop treated patients compared to $12.9 \%$ in the non-operative treated patients after 2 year follow-up. Secondary surgery was required in 5 $(11.9 \%)$ of the patients in the X-Stop group compared to 4 $(12.1 \%)$ in the control group.

Unfortunately, we experienced an extremely high failure rate, defined as surgical re-intervention, in a cohort of patients with lumbar spinal stenosis caused by degenerative spondylolisthesis treated with the X-Stop interspinous distraction device. In our cohort, the average percentage of slip was less than $25 \%$, though in 3 patients the percentage of slip was between 25 and $30 \%$. Surgical re-intervention was required in 7 (58\%) patients within 24 months. Of
Fig. 1 a Pre-operative lateral plain radiograph. b Postoperative lateral plain radiograph. X-Stop positioned at the level L4-5
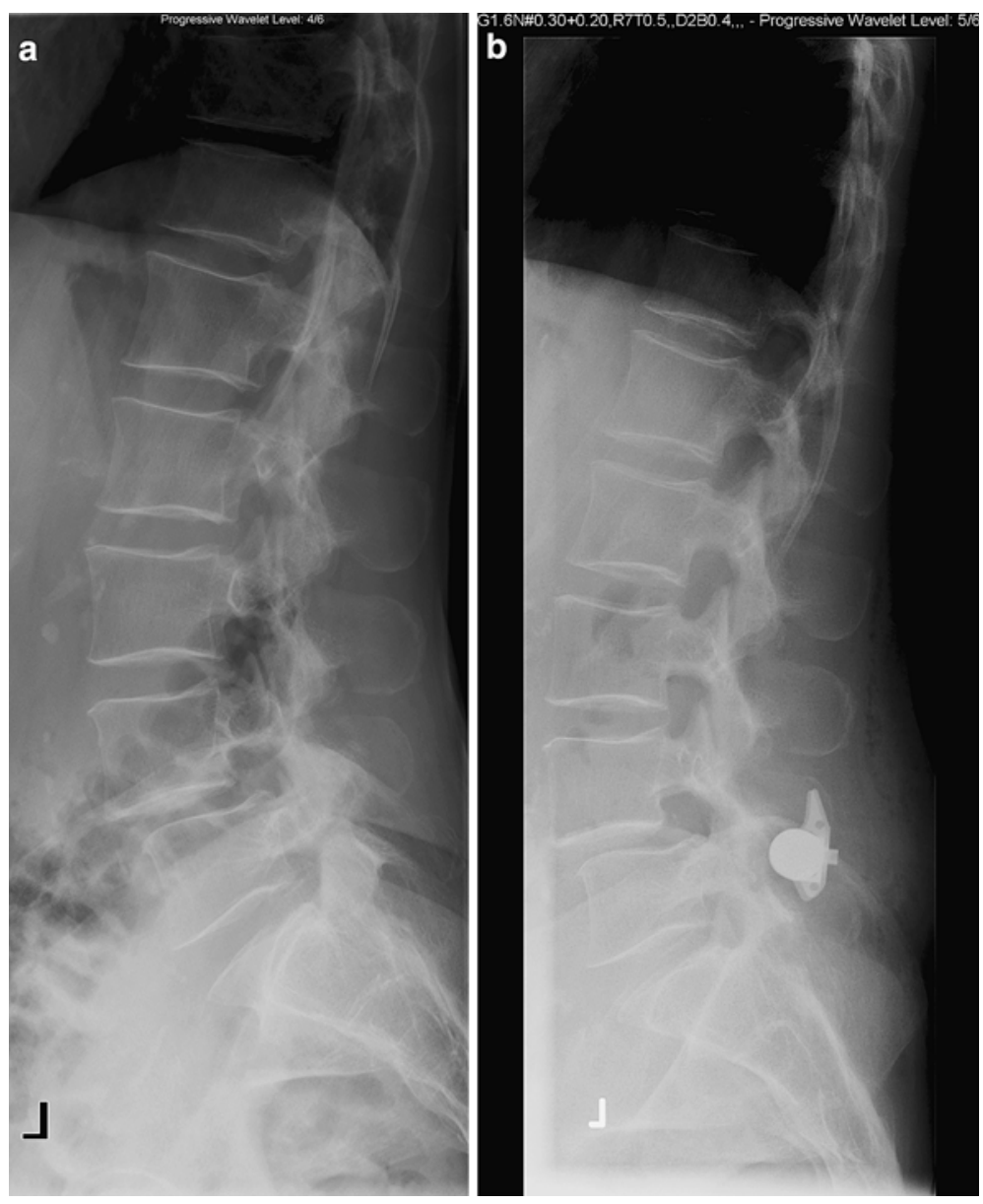

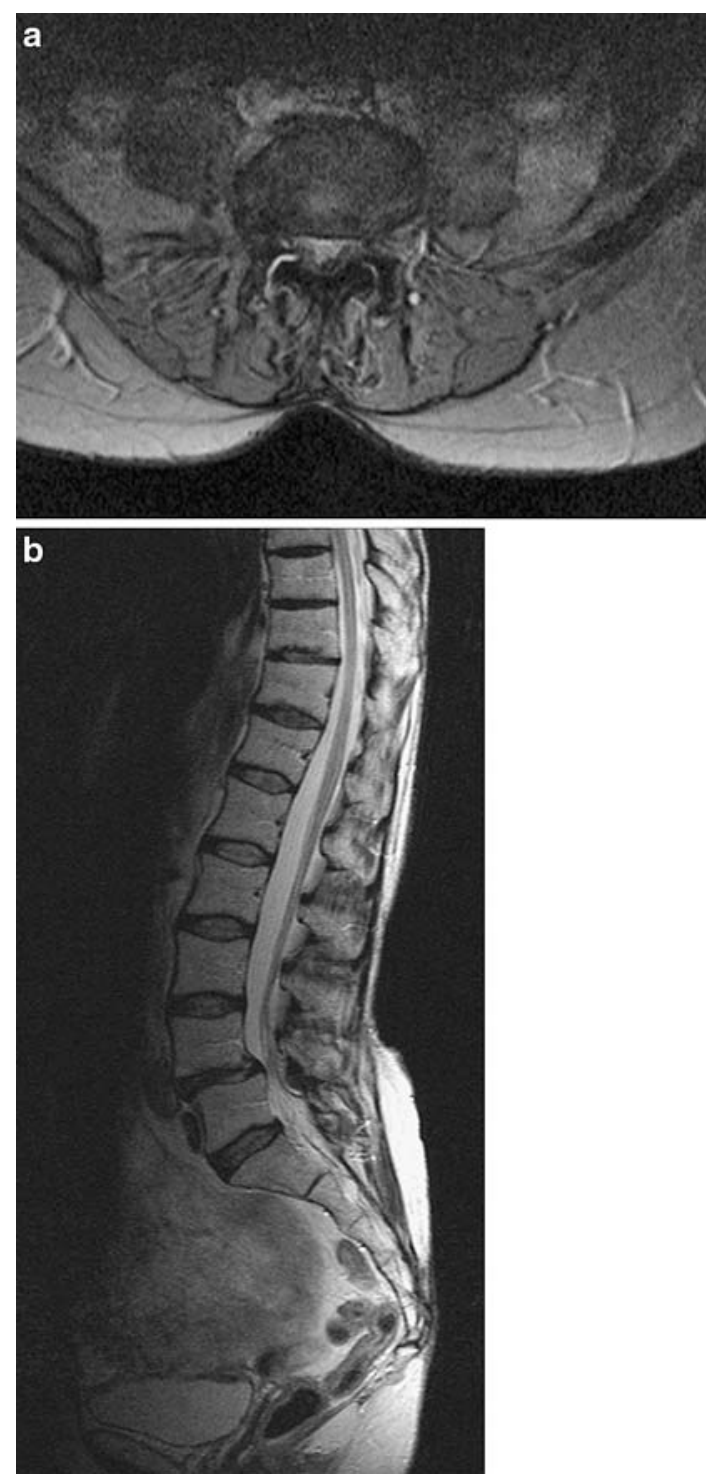

Fig. 2 Pre-operative T2-weighted a transversal and b sagittal MR Image showing lumbar spinal stenosis due to discopathy, facet arthritis, ligamentum flavum hypertrophy and anterolisthesis

these, only 1 patient had a slip of more than $25 \%$ (27.6\%). There was no relation between the severity of the slip and the failures in our cohort. Our indication for re-intervention included recurrence or persistent and unremitting low back pain and persistent or progressive neurogenic claudication with radiculopathy. Both clinical and radiological findings were considered together for diagnosing failure of treatment. Unfortunately, we did not enclose pre- and postoperative outcome measurements. However, since the surgical goal of the X-Stop include pain reduction, improvement of neurological symptoms, and improvement of quality of live, re-intervention was considered as the endpoint for failure.

In diagnosing spinal stenosis, thecal sac impingement and nerve root compression are seen on MRI. We observed
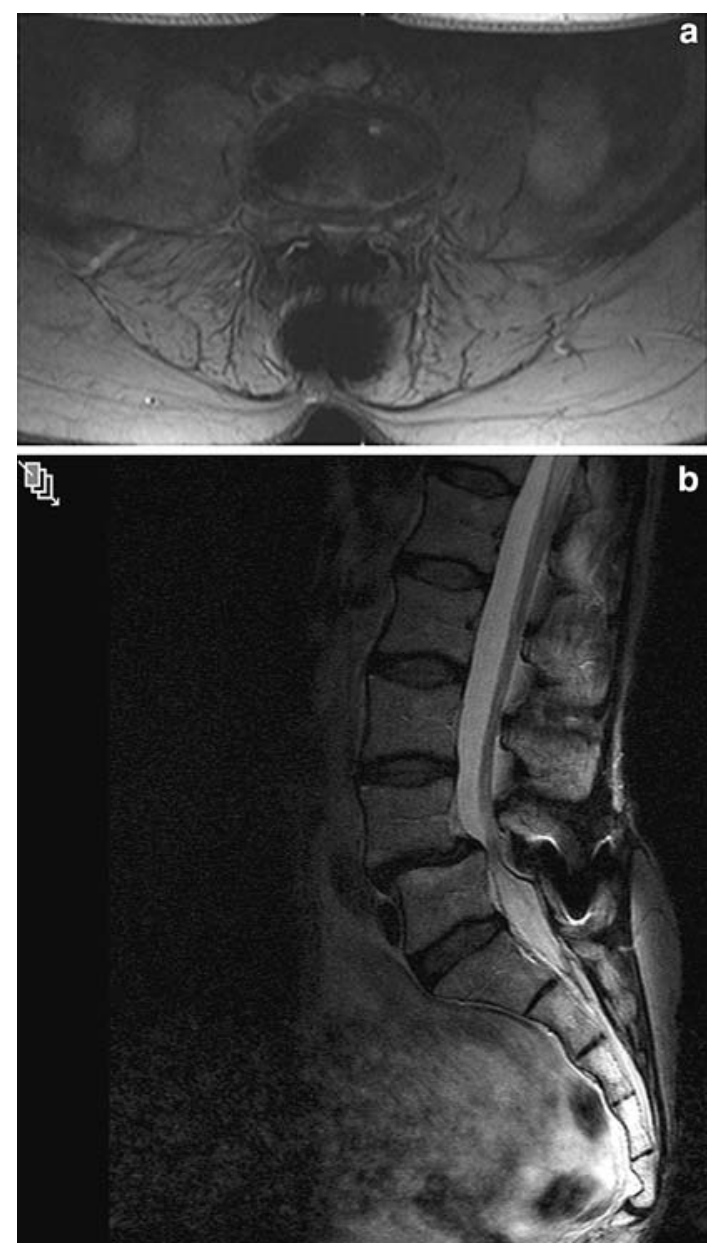

Fig. 3 Post-operative T2-weighted a transversal and b sagittal MR Image. No change in canal cross-sectional area and mid-sagittal diameter visible after insertion of the X-Stop at level L4-5

no improvement of the axial and sagittal diameter of the central canal on the MRI after insertion of the X-Stop. In addition, no relation was found between the severity of the pre-operative spinal stenosis measured on MRI and an eventual secondary surgical intervention. Recently, in a study using positional MRI pre- and post-operatively following insertion of the X-Stop, improvement of the cross sectional area of the dural sack has been observed in 12 patients with symptomatic spinal stenosis [11]. This study, however, did not include patients with spinal stenosis caused by degenerative spondylolisthesis. Unfortunately, we do not have the opportunity to use the positional MRI. It may be hypothesized that the spinal stenosis will be more severe in a standing positional MRI, as a result of the instability in degenerative spondylolisthesis. A limitation of the present study is the lack of objective standards of measurement spinal stenosis on MRI. Nevertheless, all patients in our study showed pre-operative a severe thecal sack impingement at the level of degenerative spondylolisthesis. In addition, the spondylolisthesis, as measured on 
the lateral standing radiographs, did not show progression or improvement after surgery.

From a biomechanical point of view, it may be questioned if the X-Stop interspinous distraction device provides any stabilizing effect on the affected motion segment and will increase the spinal canal in degenerative spondylolisthesis. It has been shown that the facet joints in patients with degenerative spondylolisthesis demonstrate an increased sagittal orientation [5, 7]. When the facet joints are orientated in a more sagittal plain, the resistance to shear forces is decreased. Obviously, the more sagittal orientation of the L4-L5 segment combined with an interspinous distraction device may result in a progressive forward slip of the superior vertebra, and a progressive narrowing of the spinal canal and lateral recesses. Thus, the presence of a degenerative spondylolisthesis in patients with lumbar spinal stenosis may be considered as a contra indication for the X-Stop.

In conclusion, the X-Stop interspinous distraction device showed an extremely high failure rate, defined as surgical re-intervention, after short term follow-up in patients with spinal stenosis caused by degenerative spondylolisthesis. We do not recommend the X-Stop for the treatment of lumbar spinal stenosis with degenerative spondylolisthesis, and we consider a degenerative spondylolisthesis a contraindication for the X-Stop interspinous distraction device.

\section{Competing interests}

No benefits in any form have been received or will be received from a commercial party related directly or indirectly to the subject of this article.

\section{References}

1. Anderson PA, Tribus CB, Kitchel SH (2006) Treatment of neurogenic claudication by interspinous decompression: application of the X STOP device in patients with lumbar degenerative spondylolisthesis. J Neurosurg Spine 4:463-471

2. Atlas SJ, Delitto A (2006) Spinal stenosis: surgical versus nonsurgical treatment. Clin Orthop Relat Res 443:198-207

3. Bassewitz H, Herkowitz H (2001) Lumbar stenosis with spondylolisthesis: current concepts of surgical treatment. Clin Orthop Relat Res 384:54-60

4. Christie SD, Song JK, Fessler RG (2005) Dynamic interspinous process technology. Spine 30:S73-S78

5. Cinotti G, Postacchini F, Fassari F, Urso S (1997) Predisposing factors in degenerative spondylolisthesis. A radiographic and CT study. Int Orthop 21:337-342

6. Dong G, Porter RW (1989) Walking and cycling tests in neurogenic and intermittent claudication. Spine 14:965-969

7. Grobler LJ, Robertson PA, Novotny JE, Pope MH (1993) Etiology of spondylolisthesis. Assessment of the role played by lumbar facet joint morphology. Spine 18:80-91

8. Kondrashov DG, Hannibal M, Hsu KY, Zucherman JF (2006) Interspinous process decompression with the X-STOP device for lumbar spinal stenosis: a 4-year follow-up study. J Spinal Disord Tech 19:323-327

9. Richards JC, Majumdar S, Lindsey DP, Beaupre GS, Yerby SA (2005) The treatment mechanism of an interspinous process implant for lumbar neurogenic intermittent claudication. Spine 30:744-749

10. Sengupta DK, Herkowitz HN (2003) Lumbar spinal stenosis. Treatment strategies and indications for surgery. Orthop Clin North Am 34:281-295

11. Siddiqui M, Nicol M, Karadimas E, Smith F, Wardlaw D (2005) The positional magnetic resonance imaging changes in the lumbar spine following insertion of a novel interspinous process distraction device. Spine 30:2677-2682

12. Vibert BT, Sliva CD, Herkowitz HN (2006) Treatment of instability and spondylolisthesis: surgical versus nonsurgical treatment. Clin Orthop Relat Res 443:222-227

13. Zucherman JF, Hsu KY, Hartjen CA, Mehalic TF, Implicito DA, Martin MJ et al (2004) A prospective randomized multi-center study for the treatment of lumbar spinal stenosis with the X STOP interspinous implant: 1-year results. Eur Spine J 13:22-31

14. Zucherman JF, Hsu KY, Hartjen CA, Mehalic TF, Implicito DA, Martin MJ et al (2005) A multicenter, prospective, randomized trial evaluating the X STOP interspinous process decompression system for the treatment of neurogenic intermittent claudication: two-year follow-up results. Spine 30:1351-1358 